\title{
In-Band Single-Frequency Signaling
}

\author{
By A. WEAVER and N. A. NEWELL
}

(Manuscript received June 7, 1954)

Single-frequency signaling liberates dial systems from the restrictions of dc signaling methods. This freedom, as might be expected, is most important in the long distance telephone plant where trunks are frequently too long or have no conductors for dc signaling. The general plan of signal frequency $(S F)$ signaling is based upon continuous signaling because of it's speed and reliability. In this respect it is like the usual dc trunk signaling schemes. SF uses steady current in the trunk signaling path for the normal idle trunk condition and no current in the signaling path for the other and alternate busy (talking) trunk condition. This choice of signal conditions is essential for SF signaling in-band systems, which as the name implies operate within the standard voice channel, to avoid conflict between signal and voice transmission. The same conditions are also used in SF out-of-band and separate line systems.

The in-band SF system can be used with any type or length of line facility that meets normal voice transmission requirements and is therefore the preferred method used by the Bell System to meet requirements for toll dialing on a national basis, with other signaling arrangements limited to the shorter trunks. The requirements, design considerations, main features, and method of operation for the in-band system are outlined in this paper.

\section{INTRODUCTION}

The signaling requirements for dial telephone operation are naturally more exacting than those for manual switching methods. This means a high order of signaling system is needed to satisfy the requirements for the toll telephone plant and for automatic toll switching systems described in recent papers in this JournaL. ${ }^{1}{ }^{2}$ Indeed the advantages in speed and economy of dial telephone systems depend to a large extent upon the type of signaling provided for them. The signaling arrangements for intertoll telephone trunks which are the links between telephone switching systems, therefore, become most important.

Dial operation in the past has been based upon de signaling which is 
limited to relatively short distances and to line facilities having do paths available to them. In planning for nationwide dialing of long-distance calls the need for an ac signaling system for dial telephone trunks became apparent. The length of intertoll trunks and the extensive growth in carrier line facilities which do not have associated do paths made it necessary to develop ac dial signaling systems. The single-frequency signaling plan was developed for this purpose and is the first of its kind to satisfy the conditions associated with long distance intertoll dialing in the Bell System.

There are now several trunk signaling means that may be grouped as using the SF signaling plan. These are (1) the adaptation of VF carrier telegraph requiring an additional line channel independent of the voice transmission line facilities, (2) N1 and 01 carrier signaling furnished as part of these carrier terminals using 3,700 cycles outside but adjacent to the voice paths, and (3) 1600-cycle and 2400-cycle signaling systems, the in-band systems that use the voice paths.

Both in-band and out-of-band signaling have advantages and disadvantages. The in-band single-frequency signaling system uses ac in the voice frequency range to pass full supervision and dial pulsing signals over the same paths that are furnished for voice transmission in telephone trunks. This is accomplished without any loss in band width, change in line facility or addition of intermediate signaling equipment. On most calls voice and signal transmission are not required at the same time. On the few calls going to intercept operators voice transmission is impaired slightly by the effect of signal tone being on in one direction. On calls encountering busies, it is desirable to return both flashing supervision and interrupted audible tones. This can be done with out-of-band signaling but in-band signaling can return either but not both. The signaling system allows remote build-up and breakdown and provides for supervision of the temporary connections ordinarily used. Control and supervision of distant ends of trunks is required continuously whereas dial pulsing is required only at the start of calls and speech transmission is required only when connections are established.

\section{TRUNK SIGNALS}

Before going into the details of the signaling system itself it seems appropriate to review the trunk signals it is called upon to transmit. Most intertoll trunks are arranged for two-way operation, which means that a connection can originate at either end. To permit this operation, the signaling in each direction must be symmetrical and the trunk must allow the direction in which the connection is established to determine
Table I - Calling to Called Direction

\begin{tabular}{|c|c|}
\hline Trunk Condition & Signaling Frequency \\
\hline Idle (disconnect) & On \\
\hline Connect & Off \\
\hline Dial pulsing* & $\begin{array}{l}\text { On, then off, on pulses corresponding with dial break } \\
\text { intervals }\end{array}$ \\
\hline $\begin{array}{l}\text { Ring forward } \\
\text { Disconnect (idle) }\end{array}$ & $\begin{array}{l}\text { On, then off, one pulse } \\
\text { On }\end{array}$ \\
\hline
\end{tabular}

* Multifrequency pulsing, ${ }^{3}$ a separate a-c signaling system, is a faster means of transmitting number information of ten used instead of dial pulsing. Its use eliminates only the dial pulsing signals.

Table II - Called to Calling Direction

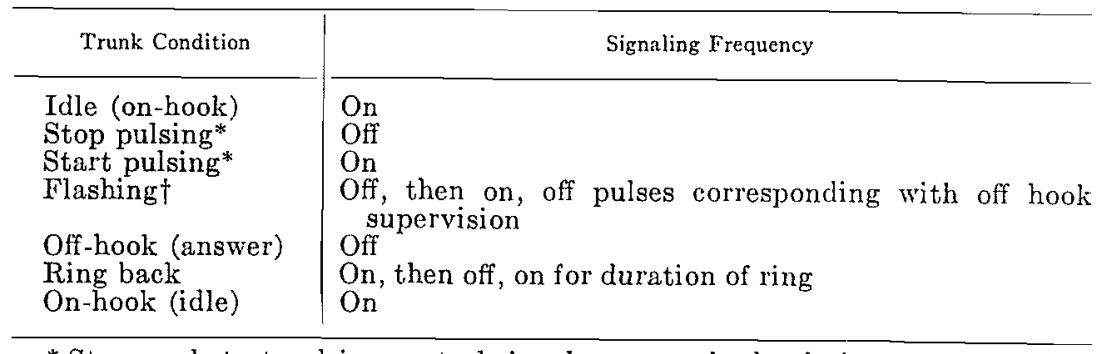

* Stop-and start-pulsing control signals are required only in connection with common control switching equipment.

t Flashing supervision signals are required only for operators; the on intervals light cord circuit lamps to inform operators of the status of calls independently of the position of cord circuit talking keys.

the signaling interpretation. The latter is conveniently identified by different names for the trunk signals in the two directions. Only two signal conditions, that is, tone on or tone off, in each direction are required for all dial trunk signals. Continuous dependence upon these two conditions assures a high degree of reliability because of signal redundancy. Tables I and II show the required dial intertoll trunk signals, together with the action taken in regard to the signaling frequency.

The signaling system must be able to handle minimum and maximum length signals. The minimum times occur in dial-pulsing where the shortest signal element may be as low as 30 milliseconds. All other types of signals have longer durations.

The maximum permissible transmission time for signals between trunk terminals is determined by the allowable unguarded intervals on twoway trunks, during which double connections may occur, and also by the stop-pulsing signal recognition interval. This time is limited to about 175 milliseconds.

The distortion permitted in the transmission of signals is proportional 
to the time duration of each signal. In general, variations in signal time should be within \pm 5 per cent. All effects of the trunk signal medium should be confined within the trunk terminals or be of such character as to have no adverse reaction in connected circuits. This is necessary for proper operation of switched connections.

\section{BASIC PLAN}

The in-band single-frequency signaling system, although fairly complex in detail, is very simple in principle. Normally, i.e., when the circuit is idle, steady tone is transmitted over the line and holds relays operated at the receiving end. Signaling is accomplished by removing and reapplying this tone, which in turn releases and reoperates the distant relays. Independent operation is obtained in each direction with one signal frequency on four-wire lines, which have separate one-way transmission pathes from terminal to terminal, and with two signal frequencies, one for each direction of transmission, on two-wire lines.

The signaling system is provided as a separate entity. It is connected in series with the transmitting and receiving branches of the line circuit at each end of a trunk and to the terminal relay circuit (trunk circuit) by two one-way signaling leads. A typical arrangement for a four-wire line terminating in a two-wire switching office at the West terminal and a four-wire switching office at the East terminal is shown in Fig. 1.

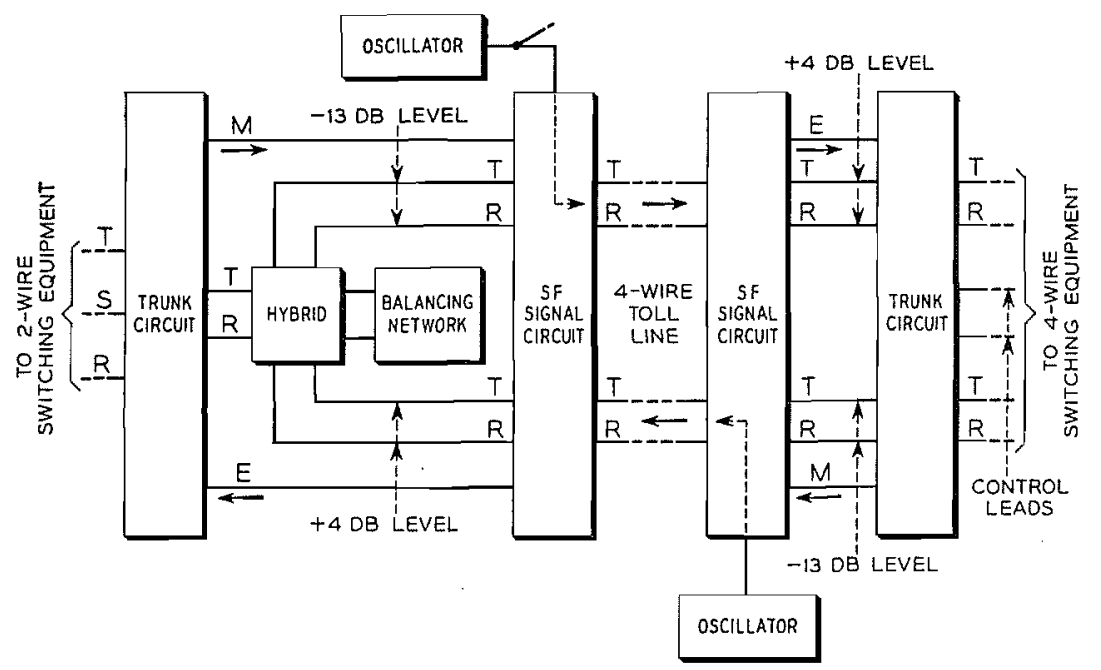

Fig. 1 - Application of single-frequency signaling to trunks with four-wire lines.
In the case of two-wire lines, the signaling equipment is applied to the four-wire transmission paths of terminal repeaters, using a different frequency for signaling in opposite directions. Band elimination networks are provided ahead of each receiving circuit to block the transmitting frequency, which would otherwise come into the receiver via echo paths and interfere with its operation.

\section{GENERAL DESIGN CONSIDERATIONS}

The successful use of the voice path for signaling, especially for continuous as contrasted to "spurt" signaling, is feasible only by a compromise among a number of conflicting factors. These factors or design considerations are (a) choice of signal frequency, (b) signaling power and receiver sensitivity, (c) imitation of signal by speech or tones, (d) interference to signal by other tones and noises, and (e) audibility of signaling tone to operators and subscribers.

\section{(a) Choice of Signal Frequency}

The choice of signal frequency is determined mainly by considerations of signal imitation by speech. As will be shown later on, signal imitation decreases rapidly as the signaling frequency is raised with the result that the highest frequency that can be reliably handled by the transmission path is used. In the case of some four-wire type lines, such as EB carrier, the highest frequency that should be used is 1,600 cycles. However, the use of 1,600 cycles results in an expensive signaling system and it is desirable to have another system using a higher frequency (2,600 cycles) for application to lines that can handle this frequency. These systems are basically the same in principle and both are described in the present article.

For application to two-wire lines the second frequency used is 2,000 cycles in the case of the older 1,600-cycle system and 2,400 cycles in the new 2,600 -cycle system.

\section{(b) Signaling Power and Receiver Sensitivity}

To limit cross talk into adjacent voice channels and to avoid adding much signal power to the repeaters it is desirable to use the lowest practicable signal power consistent with a usable signal-to-noise ratio. A value of $-20 \mathrm{dbm}$ referred to zero transmission level for the steady idle tone is satisfactory for this purpose. In order to obtain an overall margin of $8 \mathrm{db}$ the sensitivity of the receiver is set at $-28 \mathrm{dbm}$. A higher power 
is used for a short time at the beginning of each application of signaling tone to help overcome line noise and attenuation variation. This power. increase is $14 \mathrm{db}$ for the 1600 -cycle system and $12 \mathrm{db}$ for the 2,600 -cycle system.

\section{(c) Imitation of Signal by Speech or Plant Tonies}

An in-band signaling system requires that the receiver respond to signaling tone and at the same time be non responsive to speech formed currents. The principal design factors employed to achieve this feature are (1) the use of "guard action," (2) the employment of as narrow a bandwidth as practicable for the signal selective network, (3) the use of volume limiting, (4) the use of the longest operate time practicable, consistent with trunk signaling requirements, and (5) the use of the highest frequency that can be handled by the voice path.

"Guard action" is the principal means used in protecting the receiver against operation on speech. It consists in the use of nearly all frequencies in the voice band other than those in a narrow band centered on the signaling frequency to generate a voltage which is used to oppose that resulting from the signal frequency. The sum of these two voltages, plotted against frequency, for a typical receiver is shown in Fig. 2. A term used to specify the magnitude of the guard action is "guard-signal ratio" (G/S in Fig. 2) or just "guard ratio." The amount of guard which can be used is limited by signal to noise ratio because noise, like speech, tends to oppose operation of the receiver. A guard ratio in the range of 6 to $10 \mathrm{db}$ has been found to be practicable.

Protection against signal imitation is also provided by narrowing the signal frequency band as much as practicable, since this reduces the effective operating power of voice and noise frequencies. However, the extent of this narrowing is limited since the operating bandwidth must be sufficient to allow for frequency variation in the signal supply, for carrier shift in the transmission path, for variation in the elements of the tuned circuit in the receiver and to allow for the transmission of the needed side bands of the signaling pulses.

A bandwidth of 60 to 75 cycles at the $3 \mathrm{db}$ points at dialing power (about $-6 \mathrm{dbm}$ at zero level) has been adopted as about the minimum that is practicable. Because of limiting and guard action the effective bandwidth is a function of input power and in the particular designs adopted approaches about 150 cycles at the just operate point.

Volume limiting is another means used to help prevent false operation on high levels of speech. The explanation of this action is illustrated in

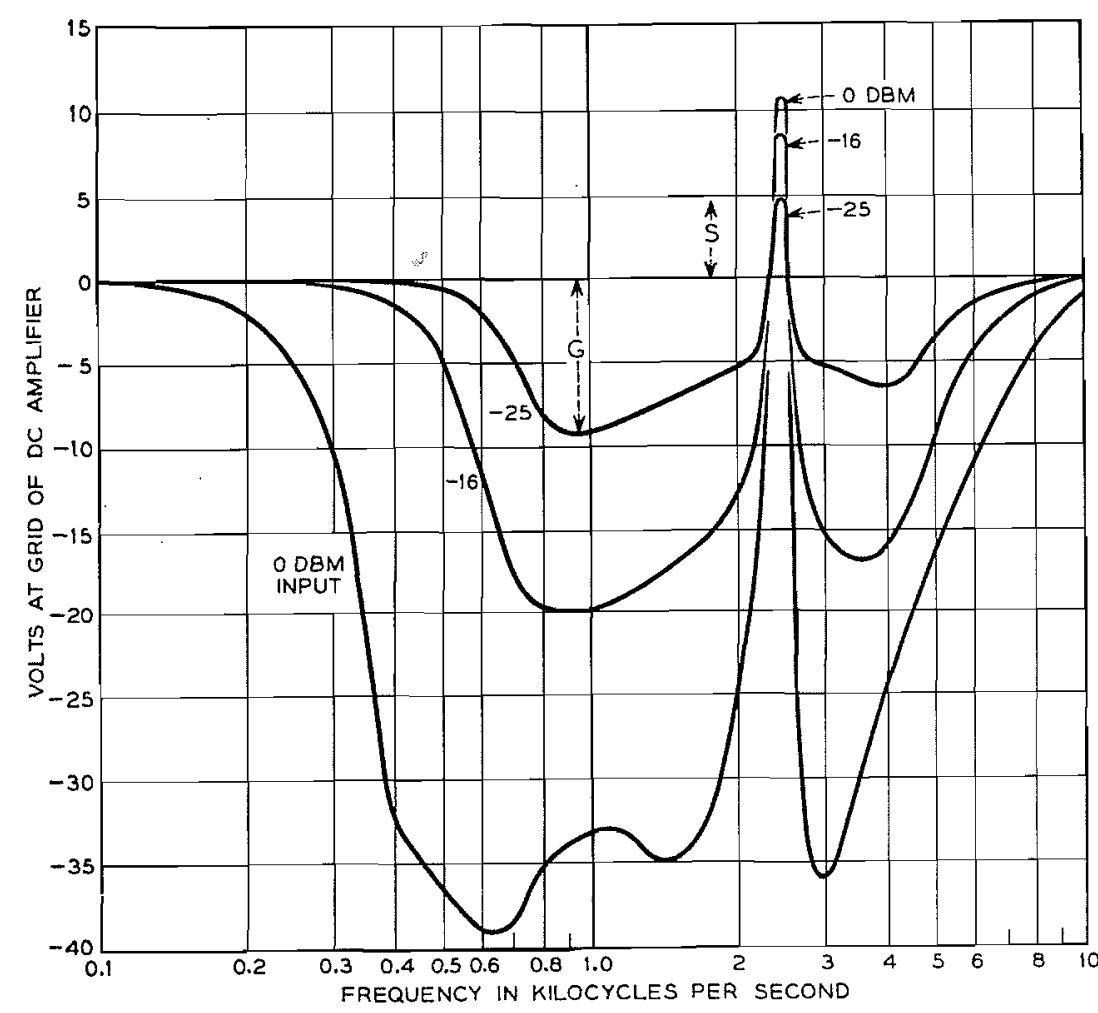

Fig. 2-Signal-guard characteristics of 2,600-cycle receiver.

Fig. 3. The dotted line shows a characteristic for a receiver with no limiting, while the solid lines are for one with limiting. As shown, a given large input would produce an output of $E_{2}$, the difference between the signal voltage and guard voltage components, for the former case and an output of $E_{3}$, which is about half as much, for the latter case. This will be less likely to operate the receiver when applied for a short interval of time, although either will produce an operation if applied long enough, because either exceeds the just operate value $E_{1}$.

Having established the basic design parameters of sensitivity, bandwidth, guard to signal ratio, limiting characteristics and speed of response it is important to know the relationship between signal imitation and the frequency used for signaling.

To obtain information on this subject a series of tests were made using a number of guard channel receivers as nearly alike as possible except for the frequencies of signal response, which were $800,1,350,1,800,2,400$ 


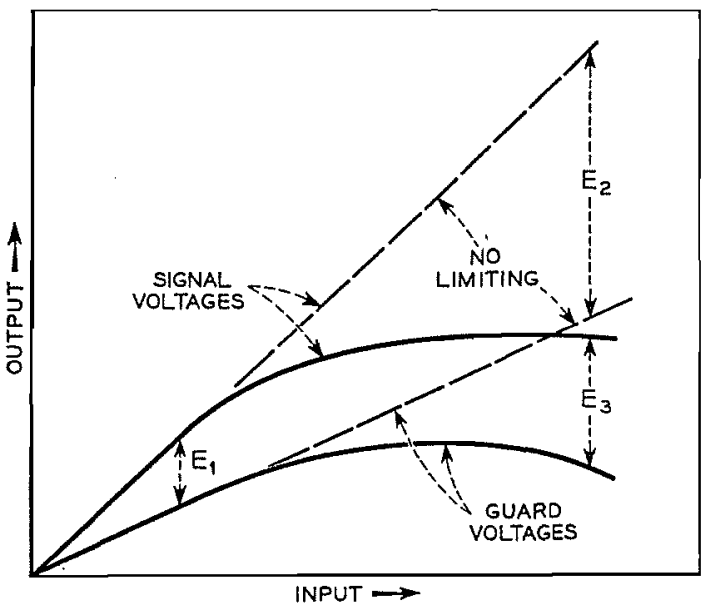

Fig. 3 - Limiter characteristics.

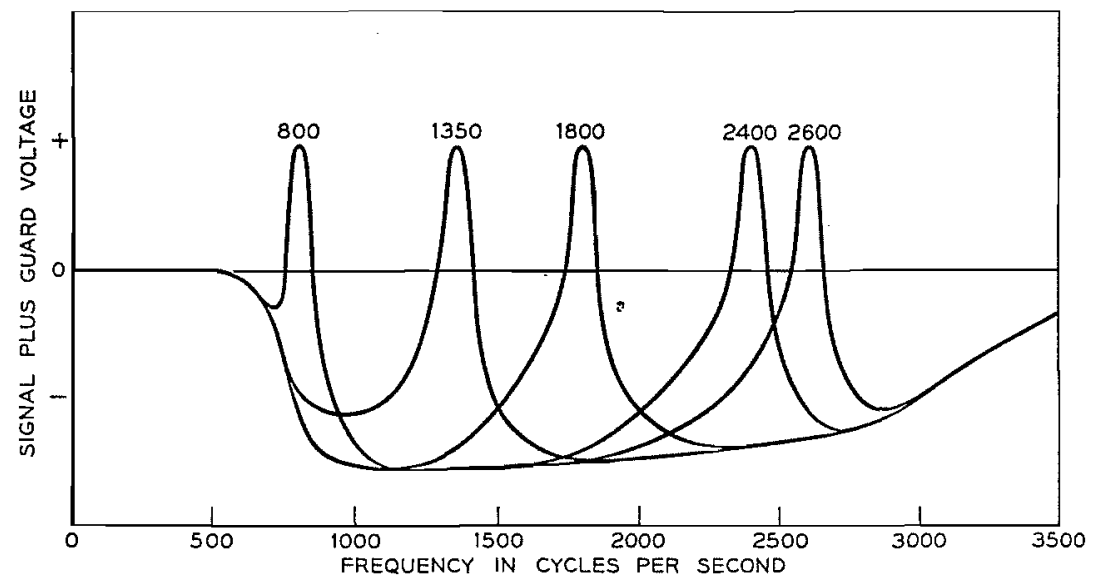

Fig. 4-Signal-guard characteristics used in signal imitation tests.

\section{TABLE III}

\begin{tabular}{|c|c|}
\hline 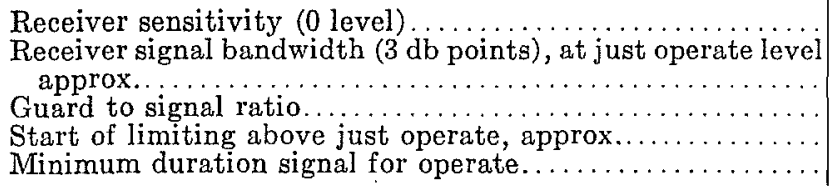 & $\begin{array}{l}-28 \mathrm{dbm} \\
150 \mathrm{cycles} \\
6 \mathrm{db} \\
5 \mathrm{db} \\
50 \mathrm{~ms}\end{array}$ \\
\hline
\end{tabular}

and 2,600 cycles. The signal-guard characteristics of all of these receivers are shown in Fig. 4. Other parameters used are given in Table III.

The results of these tests are shown in Fig. 5, where frequency is plotted against signal imitations per 100 calls. The receivers were located at New York and were connected at different times in trunks to Boston, Toronto, Buffalo, Pittsburgh, Washington and Miami, so as to get some geographical speech distribution. There was no detectable geographic effect.

The type of speech sound causing the signal imitation is also of interest even though we have not as yet been able to put this information

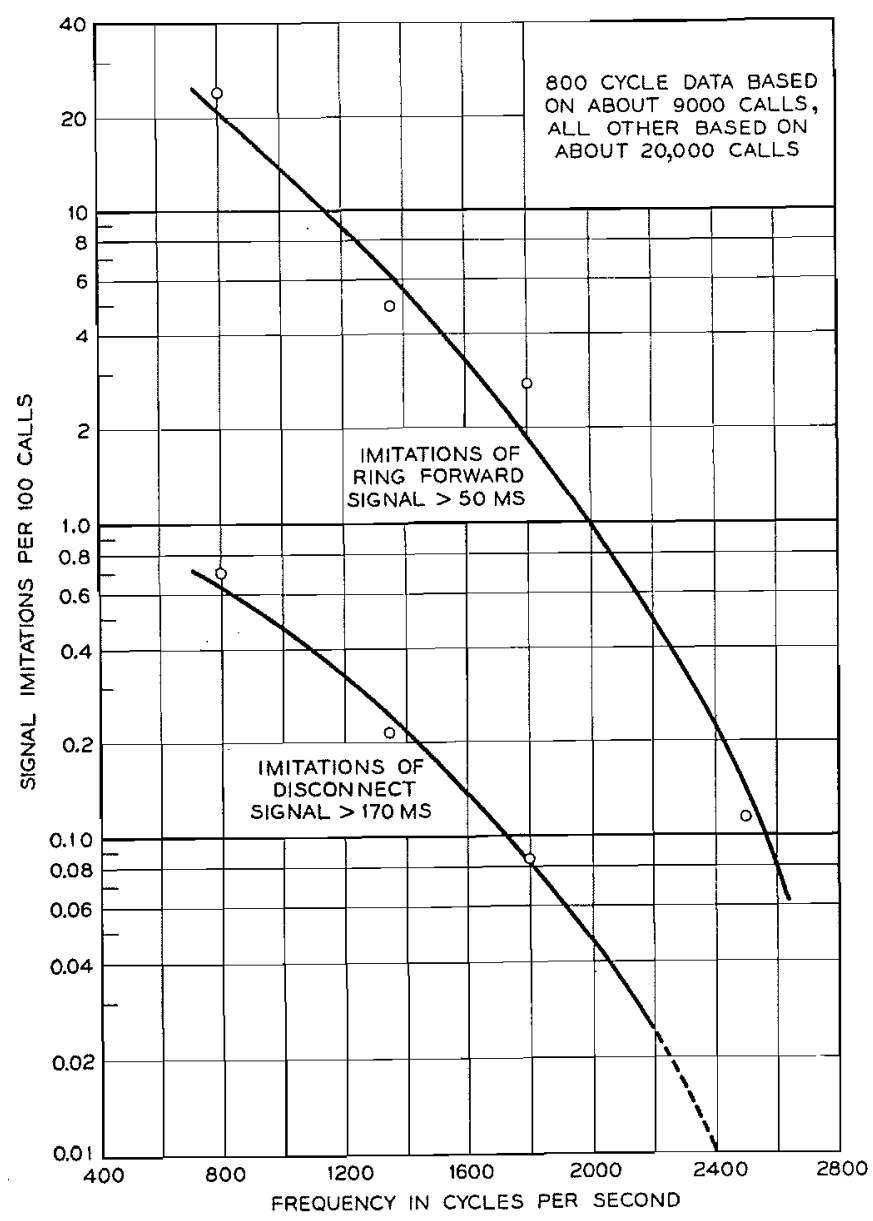

Fig. 5 - Results of signal imitation tests. 


\section{TABLE IV}

\begin{tabular}{|c|c|}
\hline 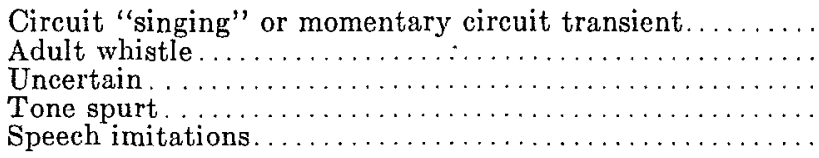 & $\begin{array}{r}10 \\
2 \\
8 \\
1 \\
48\end{array}$ \\
\hline Total. & 69 \\
\hline
\end{tabular}

to use in the design of single frequency guard type receivers. In observations on many thousands of calls it was noted that vowel type sounds were the predominant cause of signal imitations, with all except a few being formed by female speech. At the highest frequency tested $(2,600$ cycles) over 90 per cent were caused by the long $e$ vowel sound (as in feet). At the intermediate frequencies 1,350 and 1,800 cycle) most vowel sounds were noted, while at 800 cycles signal imitations were caused principally by two sounds, namely $O$ (as in hole) which accounted for about 50 per cent of the total and $a w$ (as in $a w l$ ) and similar sounds like ah as in father.

Signal imitations from vowel sounds are to be expected because of their relatively large energy and sustained nature. For instance it is well known ${ }^{4}$ that a sustained long $e$ sound can have a large component in the high frequency range with very little energy in the range from 500 to 2,000 cycles where the guard action is effective. Likewise a sustained long $O$ sound can have a large peak in the 500-cycle to 1,000-cycle range with little energy in the 1,000-cycle to 3,000-cycle range where the guard action is effective for the 800 -cycle receiver.

Speech formed currents are not the only source of signal imitation. In one series of observations using 2,600-cycle receivers involving circuits from New York to a number of other cities including Toronto, Boston, Baltimore, Washington and Miami a total of 69 signal imitations were observed. In each case an attempt was made to determine the sound that caused the false operation, with the results given in Table IV.

\section{NOISE CONSIDERATIONS}

Noise affects the signaling system in a variety of ways depending upon the nature of the noise and upon the particular signaling function being performed. When tone is first applied it is of course desired that the receiver operate. However at this time the "guard" circuit is functioning because it is also desired that the receiver be non responsive to speech. Noise, which acts on the guard circuit like speech, will therefore tend to prevent operation of the receiver. If the noise is steady and large enough it would of course permanently prevent operation, while if it is of short duration and occurred at the beginning of a signal interval it would only delay operation. Even a short delay would be harmful to ring forward or dialing signals but could be tolerated in disconnect or flashing signals.

An example of how a short duration high level noise affects receiver operation is illustrated in Fig. 6. As can be seen this particular noise transient, which happened to be caused by a relay in the trunk circuit, occurred just prior to and overlapping the tone interval. It would seriously damage a ring forward signal. The solution to this particular problem is to absorb the noise at its source, or prevent it from reaching the voice path.

After the receiver is operated for a short time ( 0.2 sec or so) the guard action is removed. Later on when the tone is removed it is desired that the receiver release, but noise at this time will tend to prevent release. The solution to this problem is a compromise in receiver sensitivity, i.e., it must be sensitive enough to hold up on the weakest signaling tone and yet release on the maximum noise that can be tolerated from a speech point of view. Fortunately such a compromise is achieved with a sensitivity that will cause the receiver to hold with the tone about $8 \mathrm{db}$
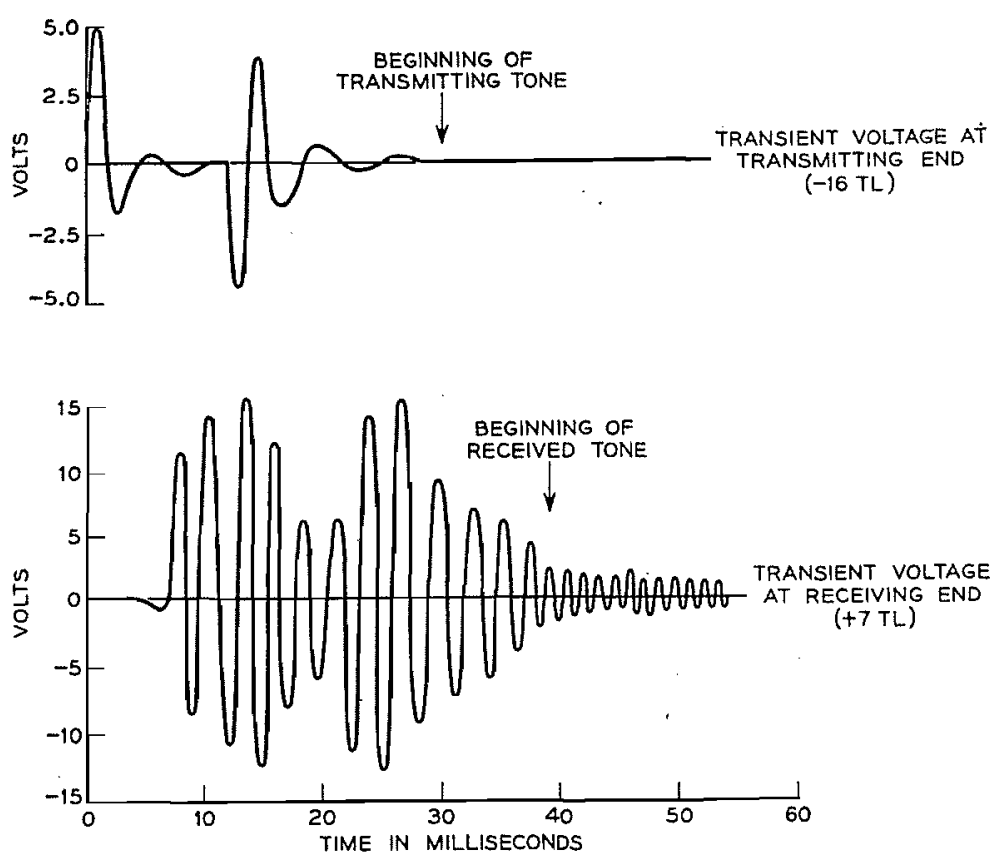

Fig. 6- Example of transient voltages generated by relay operation. 


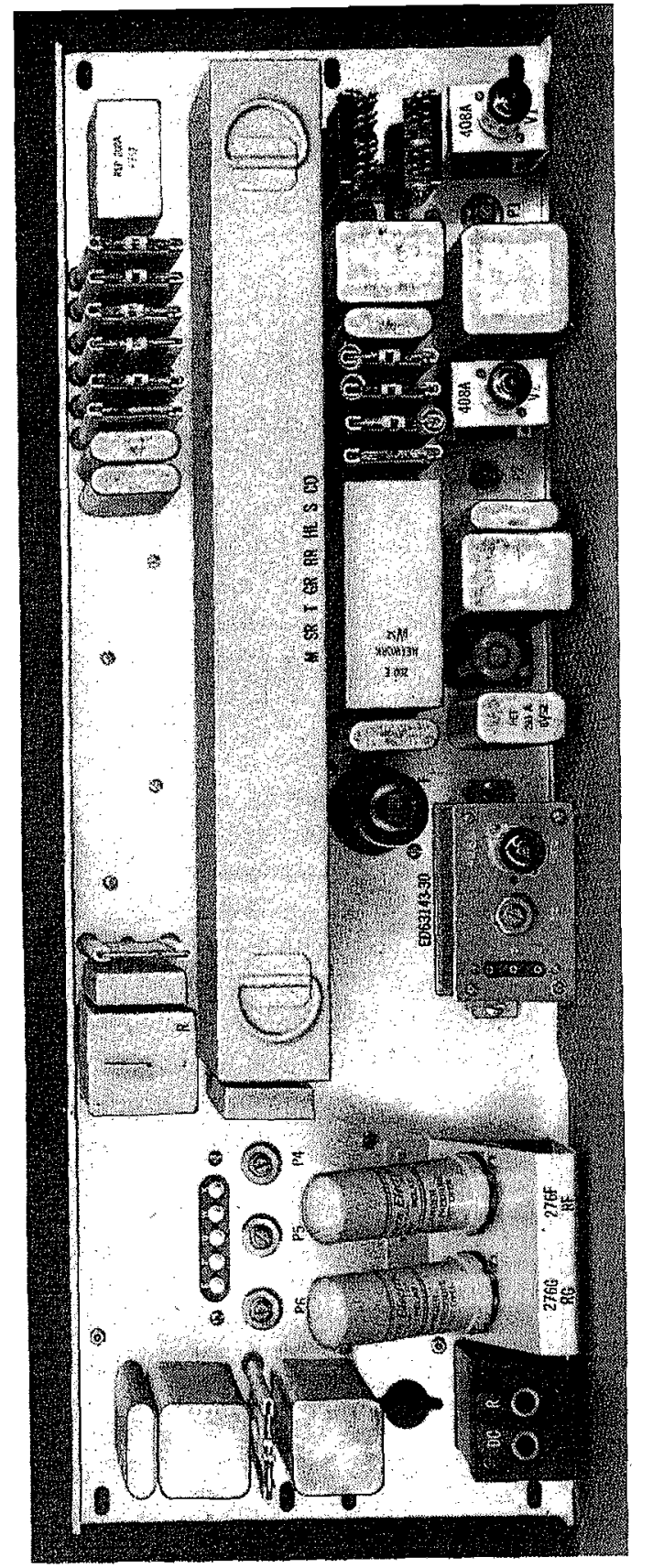

below normal and yet permit release in the presence of as much as $50 \mathrm{dba}$ of thermal noise at zero transmission level.

\section{SPECIFIC DESIGNS}

The two signaling systems 1,600 and 2,600 cycles, are basically similar in principle. However, as can be seen by reference to Fig. 5, a simple guard type receiver having a frequency of 1,600 cycles would have too many signal imitations. To overcome this the guard ratio during the talking condition was increased from 6 to $10 \mathrm{db}$, the minimum signal interval to just cause a response was increased to 100 milliseconds during the talking condition and the sensitivity was decreased to $-16 \mathrm{dbm}$. As a result fairly complicated timing and switching circuits are needed to assure that both transmitting and receiving circuits have the right condition at the right time.

Table $V$ gives a summary of the principal design parameters of the two systems.

\section{DESCRIPTION OF 1,600-CYCLE DESIGN}

A front view of the 1,600-cycle main unit is shown in Fig. 7. This panel is 8 inches high by 23 inches wide and weighs about $20 \mathrm{lb}$. The essential elements of the circuit are shown in Fig. 8. It connects to the trunk relays over two leads $\mathrm{E}$ and $\mathrm{M}$ and into the line circuit via leads labeled $T, R, T_{1}$ and $R_{1}$. The transmitter, shown in the upper portion of the figure, uses dc biased germanium varistors (diodes) to control the application of signal current to the line, and for control functions, uses four relays designated $\mathrm{M}, \mathrm{CO}, \mathrm{HL}$ and RR. The functioning of the first three except for tone control are described under the heading "Description of 2,600 Cycle Design" later in this article. The RR relay (not shown) in conjunction with the $\mathrm{M}$ relay lengthens the sent pulse for the ring for-

TABLE V

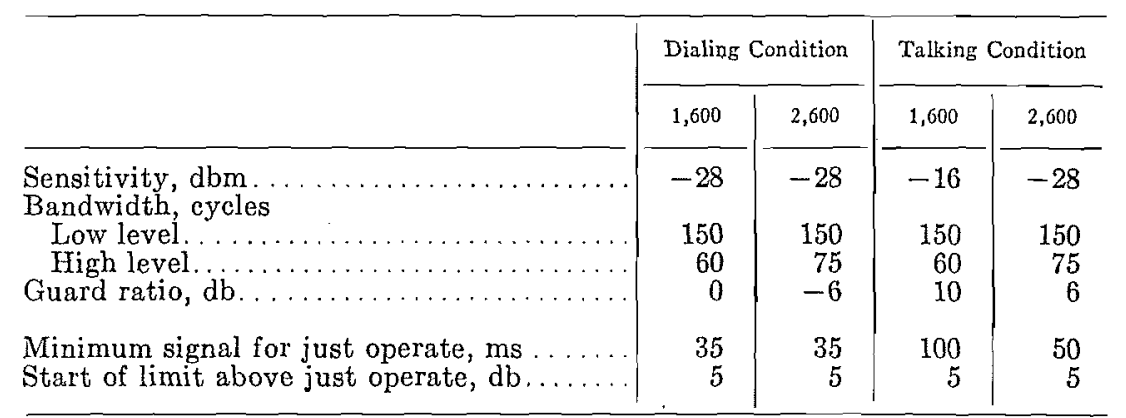




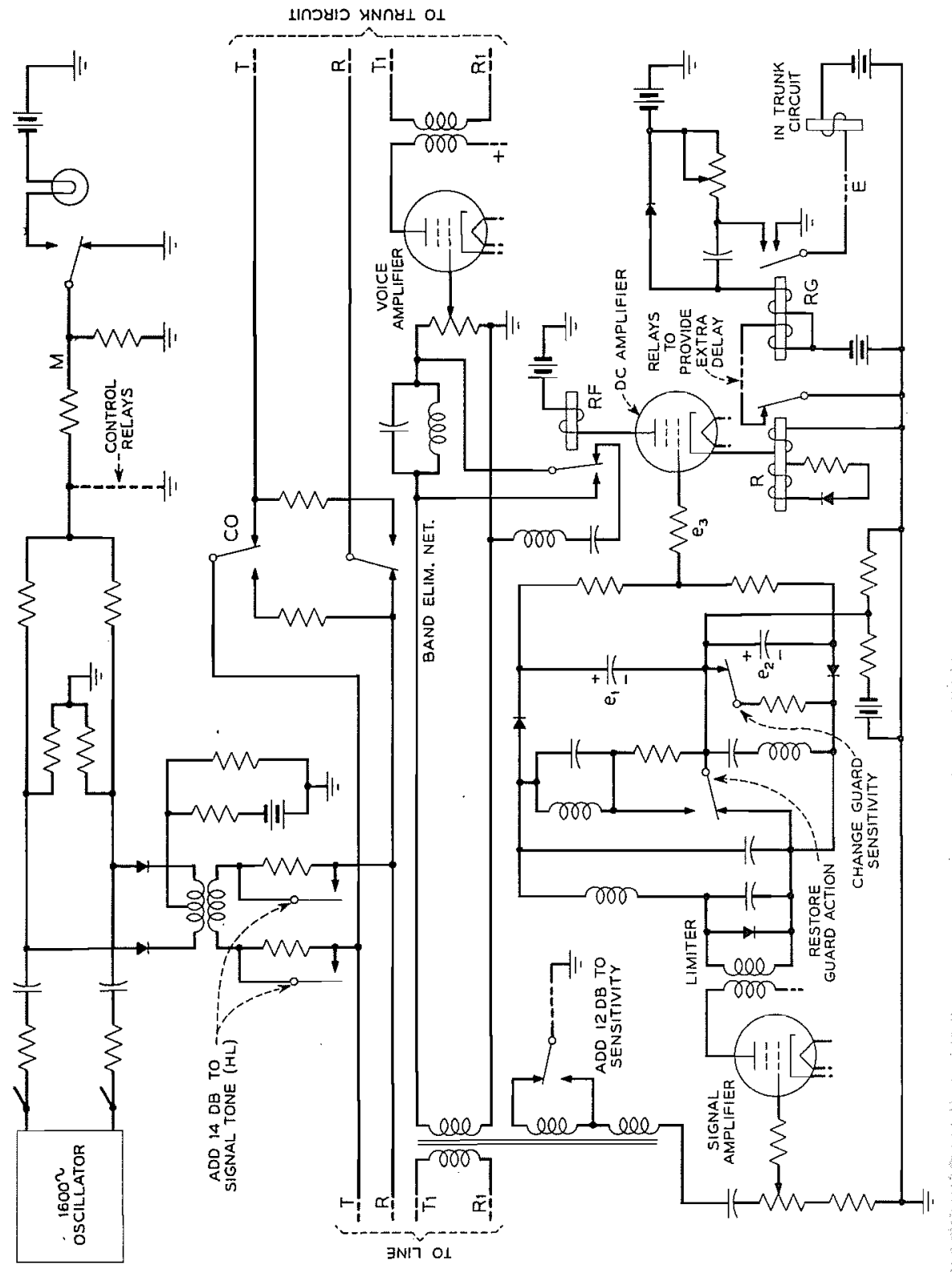

Fig. 8-Simplified diagram of 1,600-cycle signaling circuit. ward signal because the far end receiver at this time has a long operate time.

The signal receiver is connected in series with the receiving branch of the voice transmission path and is provided with a voice amplifier to provide a blocking function so noises originating in the switching equipment or beyond will not interfere with operation of the signaling receiver, and to compensate for the signaling bridging loss.

The receiving portion of the circuit is shown in the central and lower portions of Fig. 8. The idle condition of the trunk is shown, tone is being received, the $R, R G$ and $R F$ relays are operated and the band elimination filter is inserted in the receiving branch to prevent signaling tone from entering a connected circuit and interfering with signaling there.

The signal currents coming in from the line are passed through the signal amplifier, limiter and low pass filter and applied to the signalguard network from which signal voltage is applied to the dc amplifier tube to operate the above mentioned relays and open the $E$ lead, which extends into the trunk circuit. Typical wave forms at several points in the circuit are shown in Fig. 9. The extra operate time provided during the talking condition is obtained from slow relays (not shown) which at this time are in the path from the $\mathrm{R}$ to the $\mathrm{RG}$ relay. These relays also change the sensitivity, and guard ratio.

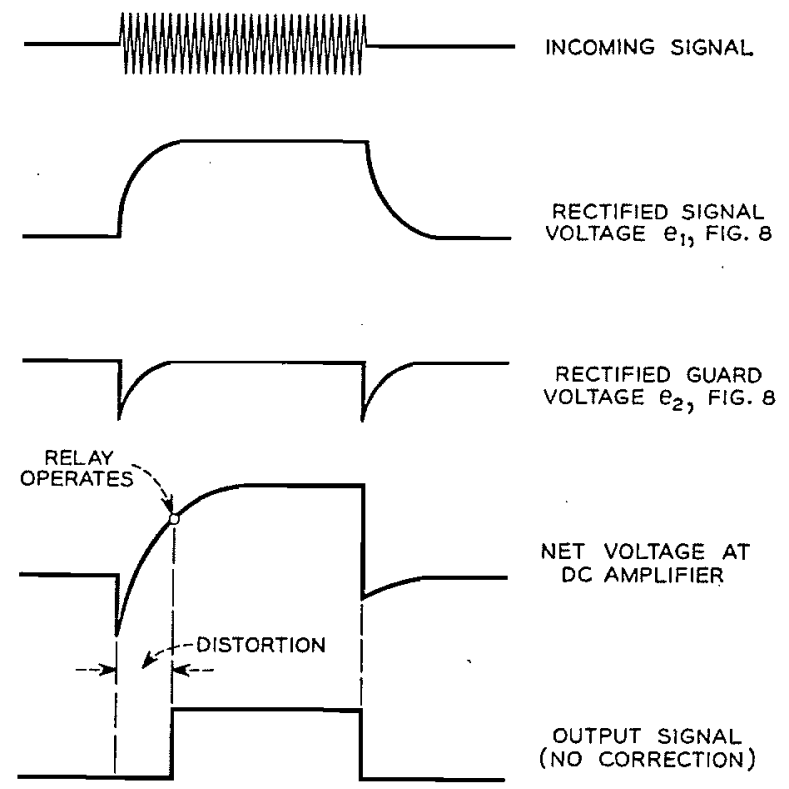

Fig. 9 - Typical wave forms in 1,600-cycle receiver. 


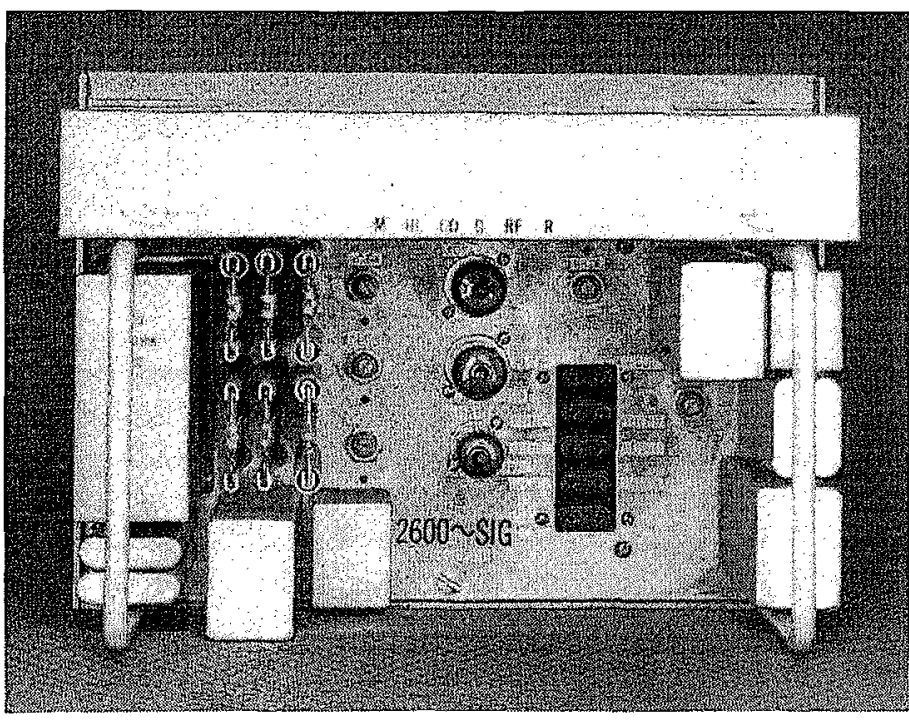

Fig. 10-Front view of the 2,600-cycle signaling panel.

The $R$ delay, because of guard action and the fact that its secondary winding is closed through a varistor and resistance, is relatively slow to operate and fast to release. For this reason some form of pulse correction is necessary to get good dial operation. This is obtained with the RG (regenerate) relay and its associated $\mathrm{CR}$ timing network, and there results an output pulse within the needed limits, even though the signal on the $R$ relay is shortened considerably.

\section{DESCRIPTION OF 2,600-CYCLE DESIGN}

The 2,600-cycle unit, shown in Fig. 10, is just half the size of the 1,600cycle unit, costs less than half as much, and is of the "plug in" type so it can be readily replaced for maintenance action.

A simplified diagram of the new signaling circuit is shown in Fig. 11, with the transmitting portion in the upper part of the figure and the receiver in the lower part. The transmitting portion employs three relays designated $\mathrm{M}, \mathrm{HL}$, and $\mathrm{co}$ which are interconnected to perform the following functions: relay $\mathrm{M}$ is used to key the signaling tone; relay $\mathrm{HL}$ (high level) adds 12 decibels to the tone power at the beginning of each signal tone application to improve signal reliability in the presence of line noise and variations in attenuation; and relay co (cut off) cuts the line momentarily to prevent noises originating in the switching equipment from interfering with signaling.
The operation of the receiver will be explained by describing its action (except for pulsing which will be described later) when signal frequency is received. This ac tone is amplified (or limited if it is too large) and then passed on to the signal and guard networks where a relatively high voltage results in the signaling channel and a lower voltage in the

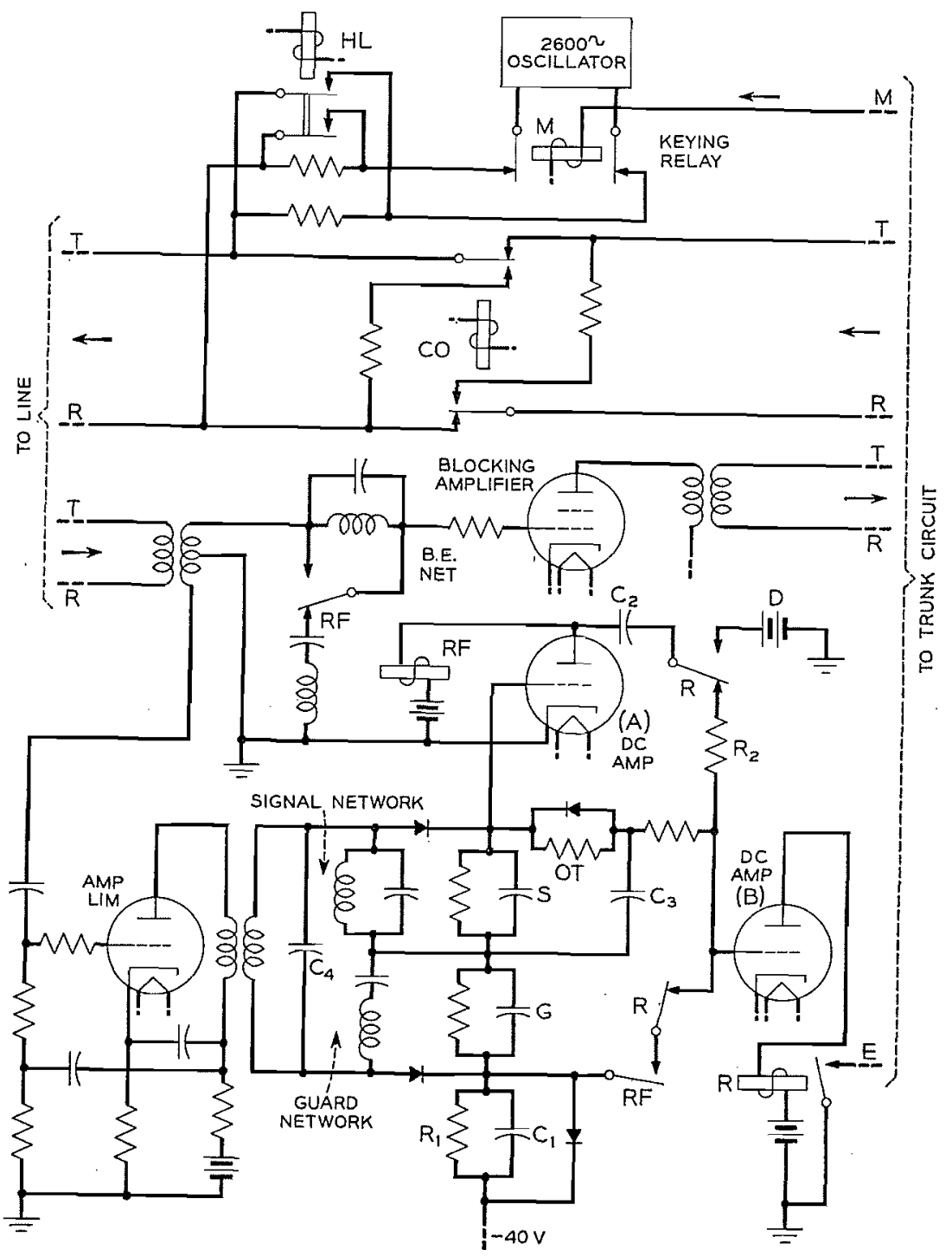

Fig. 11 - Simplified diagram of 2,600-cycle signaling circuit. 
guard channel. These voltages are then applied to rectifying circuits where positive and negative dc voltages are developed and passed on to the de amplifier tubes $\mathrm{A}$ and $\mathrm{B}$, respectively. The $\mathrm{RF}$ relay operates first and cuts in the band elimination filter thereby preventing signaling tones from entering a connected toll line and interfering with the signaling there. However, a short spurt of tone will get through because of the finite time required to operate this relay, and the $\mathrm{R}$ relay must therefore be made slow enough so that it will not operate on this tone. This action is obtained with the resistor-capacitor network (oT and $C_{3}$ ) in the grid circuit of the associated de amplifier.

A short time (about $200 \mathrm{~ms}$ ) after the $\mathrm{R}$ relay operates, a relay (not shown) releases, which short circuits the guard network and inserts enough resistance in series with the signal network to substantially remove its tuning. The purpose of removing the guard action during the idle condition is to prevent release of the receiver which would otherwise be caused by occasional bursts of line noise.

The signal network is made broad at this time for the following reason. In connections to an intercepting operator, "off hook" supervision is not returned to the originating end to avoid charging for the call. This means that tone remains on the line to continue to hold up the receiver. At the same time the intercepting operator's speech must of course be transmitted over the line so that both speech and tone enter the receiver.

Speech can be of a relatively high power as compared to the tone with the result that the action of the limiter tends to suppress the tone and could falsely release the receiver if the signal tuning were present. However, with broad tuning either speech or tone will hold up the receiver and no trouble is encountered.

The blocking amplifier seen in Fig. 11 has the same function as in the 1,600 -cycle design described previously.

Among the new features in this unit, one of the most significant is the pulse-correcting circuit. This feature is a very important element in the entire long distance connection since it serves to keep the length of the dial pulses within specified time limits. The dial pulses on many calls may have to go through a number of central offices and all their associated equipment, and in each stage of the transmission path the ideal 60 -millisecond dial pulse may be distorted so that it becomes too long or too short.

The pulse-correction is accomplished by generating appropriate transient voltages, whose duration is determined by capacitor-resistor networks. These voltages are then applied to the grid of dc amplifier $\mathrm{B}$ in Fig. 11 to perform the elongation or shortening of the pulses as required.
Dial pulses of 2,600-cycle tone with various degrees of distortion enter the circuit at the left of the diagram. It, is desired that they be corrected, converted to an interrupted dc current in lead $\mathrm{E}$ by means of relay $\mathrm{R}$, and then passed on through the toll office. This break in the dc current will effectively key the next outgoing tone transmitter, which faithfully reproduces the break intervals. The $R$ and RF relays are shown operated as would be the case if an originally short dial tone pulse were being converted into a longer dc current break in lead $\mathrm{e}$, i.e., closer to 60 milliseconds in duration.

Currents of signal frequency build up voltages across the "signal network," while currents of any other frequency, such as those from speech, build up voltages across the "guard network." These voltages are rectified separately, as shown, to obtain oppositely poled do voltages across the signal capacitor s and the guard capacitor $\mathrm{G}$. During normal speech, the voltages across the guard capacitor will dominate and, being negative, will keep the grid of dc amplifier $\mathrm{B}$ negative to prevent operation of the $\mathrm{R}$ relay at this time.

However, when dial tone pulses are applied, the main voltage will appear across the signal capacitor, with a small transient voltage appearing across the guard capacitor due to sidebands of the dialing frequency. This transient, being negative, produces an effect shown at (a)
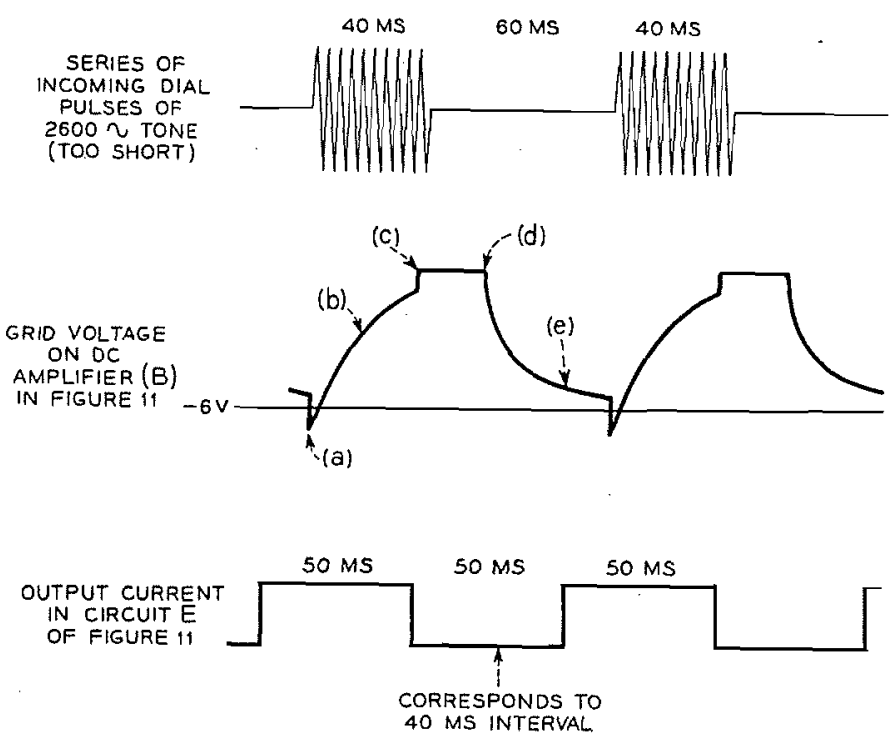

Fig. 12 - Wave forms for lengthening of short dial pulses. 
in Fig. 12, the middle diagram of which represents the voltage on the grid of the dc amplifier $\mathrm{B}$. The voltage on the grid of this amplifier will thereafter build up slowly as shown at (b) because of the or (operate time) resistor and $C_{3}$ capacitor. In this illustration it is assumed that the incoming dial pulse has a duration of only 40 milliseconds, which is shorter than desired. In this particular case the RF relay operates about at time (b) and the $\mathrm{R}$ relay at point (c), which is just prior to the end of at time (b) and the $\mathrm{R}$ relay at point (c), which is just prorin a corrected output the pulses. It is the release of the $\mathrm{R}$ relay. This is accomplished by causing signal, to delay the release of the $\mathrm{R}$ relay. Thisier tube $\mathrm{B}$ from time (c) to time (d). This current comes from energy stored in $\mathrm{C}_{2}$ from battery $D$ time (d). This current comes from energy stive transient at the plate of tube $A$ grionerated by the end of the pulse. At time (d) the RF relay releases, and the voltage on the grid of the dc amplifier $B$ decays along the line from (d) to (e), at which point relay $\mathrm{R}$ releases. The result is that the $\mathrm{R}$ relay has sent on a pulse that has been corrected from an original 40 to a final 50 milliseconds. It is possible but uneconomical to build a unit that would achieve perfect pulse correction to 60 milliseconds, but when a number of toll lines are in tandem, the pulses will have to pass through

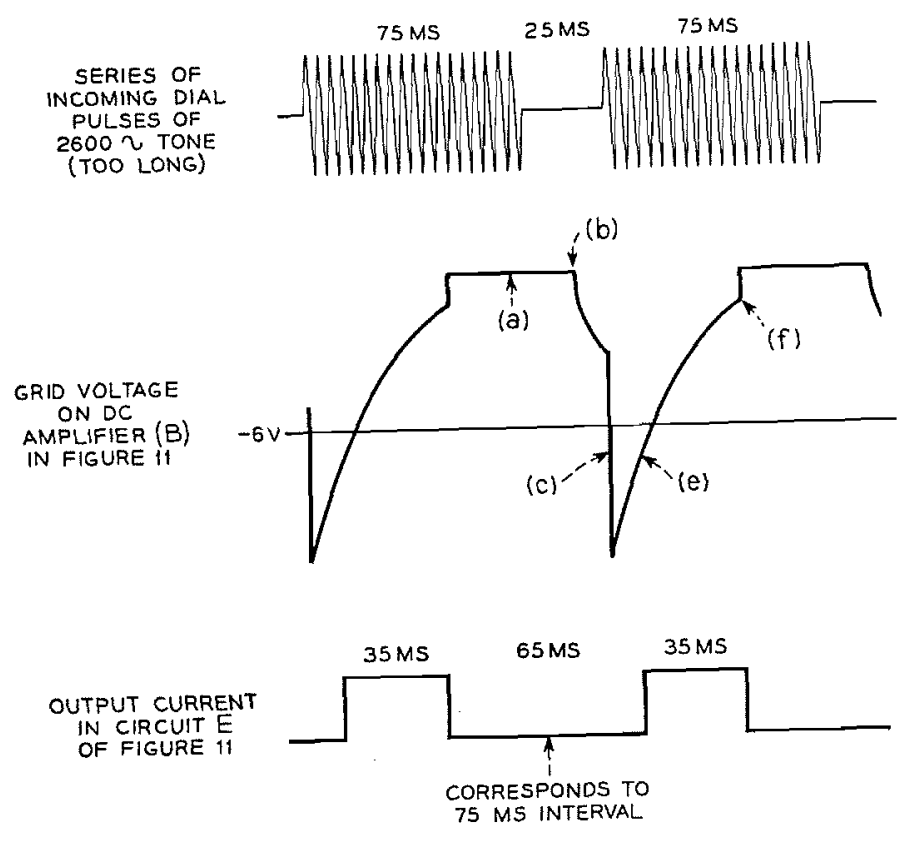

Fig. 13 - Wave forms for shortening of long dial pulses. several SF units, and the pulses will therefore be brought close to 60 milliseconds by the successive corrections.

The action of the pulse corrector when the incoming signal is too long will be explained with the help of Fig. 13. Here the cycle of events will be assumed to start at (a), just at the end of an incoming dial pulse. From (a) to (b) current will flow into the grid of the dc amplifier B, at (b) the $\mathrm{RF}$ relay will release, and the voltage will start to decay, all actions so far being the same as in Fig. 12. In this instance, however, a new pulse would come along before the $\mathrm{R}$ relay has had a chance to release, and unless something is done about it, it would not release at all.
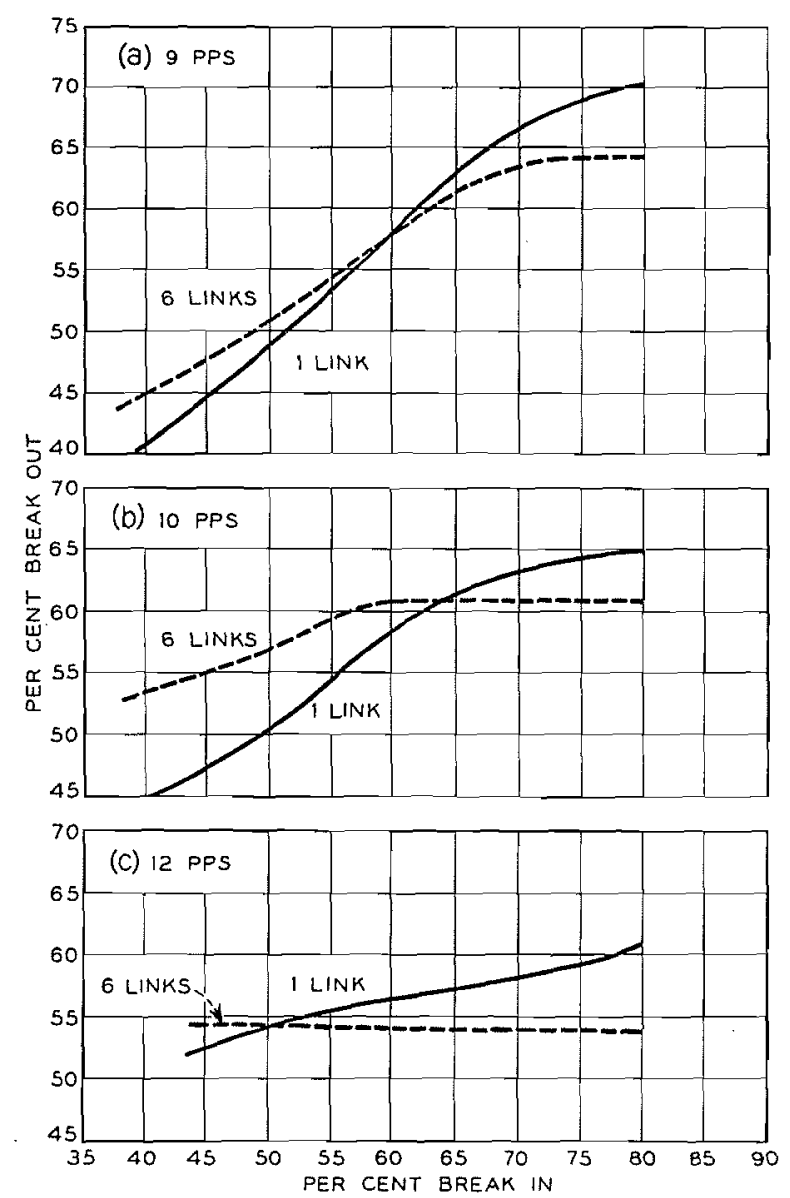

Fig. 14 - Per cent break input versus output characteristics for (a) 9 pulses per second, (b) 10 pulses per second, and (c) 12 pulses per second. 
This "something" consists of using the large negative transient voltage generated at the plate of tube A resulting from the application of a positive pulse to its grid. This transient, shown at (c) drives the grid of $\mathrm{B}$ rapidly and heavily negative, thereby forcing the $\mathrm{R}$ relay to release. The remainder of the pulse-correcting action consists in using this same transient to delay the reoperation of the $\mathrm{R}$ relay. This is accomplished by storing some of the energy in the $R_{1} C_{1}$ network. This slows the building up of the voltage as shown at (e), and the relay $\mathrm{R}$ reoperates at (f). The resultant repeated signal is shown below, where a 75 -millisecond signal has been pulse-corrected to 65 milliseconds, further corrections being effected in the subsequent SF units.

Dialing performance of typical signaling units is shown by graphs in Figs. 14(a), (b), and (c). These curves show per cent break input plotted against per cent break output for 9,10 and 12 pulses per, second for one and 6 -link operation. If the system were linear the input-output characteristic would be a 45 degree straight line. When the slope is less than 45 degrees there is pulse correction, and if the slope were zero with an output at 60 per cent, pulse correction would be perfect. It is noted that the pulse correction action improves as the speed increases and at 12 pulses per second the output is nearly independent of input.

\section{ACKNOWLEDGEMENTS}

The success of this project is the result of contributions by many people, and all cannot be named specifically. However special mention should be made of $F$.A. Hubbard, who designed the equipment arrangements of the 2,600-cycle system and W. W. Fritschi, C. W. Lucek, R. O. Soffel and A. K. Schenck who made important contributions to the circuit design.

\section{REFERENCES}

1. J. J. Pilliod, Fundamental Plans for Toll Telephone Plant, B.S.T.J., 31, pp. 832-850, Sept., 1952.

2. F. F. Shipley, Automatic Toll Switching Systems, B.S.T.J., 31, pp. 860-882, Sept 1952

3. C. A. Dahlbom, A. W. Horton, Jr., and D. L. Moody, Application of Multifrequency Pulsing in Switching, A.I.E.E. Transactions, 68, pp. 392-396, 1949

4. H. Fletcher, Speech and Hearing, Van Nostrand.

\section{Centralized Automatic Message Accounting System}

\author{
By G. V. KING
}

(Manuscript received May 6, 1954)

A centralized automatic message accounting system (CAMA) has been developed so that the billing data can be recorded at a ceniralized crossbar tandum office for message unit and toll calls originated by telephone customers served by a large number of local dial central offices. It is an essential part of facilities for economical nationwide customer dialing through central offces with older types of switching equipment and through other central offices which could not otherwise economically give this service. The new system records the billing data on paper tapes in the same form now used by local automatic message accounting systems. Tapes for both local and centralized automatic message accounting systems are processed in the same accounting center.

\section{INTRODUCTION}

One broad objective of the Bell System is to extend the customer's dialing range so that ultimately he will be able to dial his own calls to any telephone in the country in much the same way as he now dials local calls. Several steps toward this goal have already been taken. A revised fundamental plan $^{1}$ for automatic toll switching ${ }^{2}$ has been adopted which involves among other things the use of a nationwide numbering plan ${ }^{3}$ covering the United States and Canada. In accordance with this plan each customer will be given a distinctive 10-digit designation which will consist of a 3-digit regional or area code, a 3-digit central office code, and a 4-digit customer's number. In many parts of the country automatic toll switching systems ${ }^{4}$ are now in use by operators who complete more than 40 per cent of all toll calls by dialing directly to the called telephone in distant cities.

In order that customers may use these switching systems to dial their own toll calls, some automatic means for recording the necessary billing information on such calls must be provided. 